\title{
Multiloop Bubbles for hot QCD
}

\author{
E. Bejdakic ${ }^{\mathrm{a}}$ \\ ${ }^{\text {a }}$ Fakultät für Physik, Universität Bielefeld, 33501 Bielefeld, Germany
}

\begin{abstract}
In this article, we present analytical expansion results of two single mass scale four-loop vacuum integrals in $d=3-2 \epsilon$ dimensions. After finding hypergeometric representations with halfinteger coefficients, we use algorithms which we implemented in FORM to expand these in terms of nested sums.
\end{abstract}

Key words: Finite temperature, multiloop calculation, hypergeometric function, expansion PACS: $11.10 . \mathrm{Wx}, 12.38 . \mathrm{Bx}$

\section{Introduction}

The series of the pressure of QCD in weak coupling expansion is not analytical in the coupling $g^{2}$. The reason for this is that hot QCD is a multiscale system. One way of dealing with this problem is using effective field theory approach [12]3. In this approach the different scales are separated within different effective theories. At temperatures well above $T_{c}$ all the relevant information of QCD is contained within a three dimensional effective field theory, the so-called EQCD. For the specific problem mentioned above, EQCD has the following set of master integrals up to four loops 4, obtained using methods from [51617:

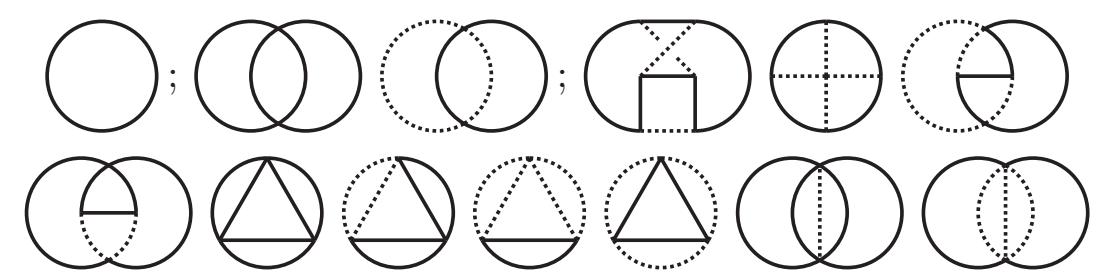

The full/dotted lines stand for a massive/massless scalar propagators. These Masters need to be computed. There are several methods for doing this; among the most frequently 
used are solving the corresponding difference equations [7, as well as the Mellin-Barnes method [89]. Here we will only be using Mellin-Barnes method for obtaining hypergeometric representation of some Masters, while as a second step we use nested sums [10[1] for expanding these hypergeometric functions analytically around $d=3-2 \epsilon$ dimensions.

\section{Mellin-Barnes method}

The Mellin-Barnes method for evaluating massive Feynman integrals consists of writing down a massive propagator in terms of a massless one 8 :

$$
\frac{1}{\left(k^{2}+m^{2}\right)^{\beta}}=\frac{1}{\left(k^{2}\right)^{\beta} \Gamma(\beta) 2 \pi i} \int_{-i \infty}^{i \infty} d s\left(\frac{m^{2}}{k^{2}}\right)^{s} \Gamma(-s) \Gamma(\beta+s),
$$

where the contour of integration is chosen such that the poles of $\Gamma(-s)$ are to the right of the contour. One simple example is the one-loop integral of eq.(3), which using the Mellin-Barnes method gives us a massless one-loop integral which in turn can be evaluated in terms of Gamma functions. At the end one can perform the complex Mellin-Barnes integral closing the contour to the right, obtaining:

$$
\begin{aligned}
\int \frac{d^{d} p}{\left(p^{2}\right)^{a}\left((k-p)^{2}+m^{2}\right)^{b}}= & \pi^{\frac{d}{2}}\left(m^{2}\right)^{\frac{d}{2}-a-b} \frac{\Gamma\left(\frac{d}{2}-a\right)}{\Gamma(a) \Gamma(b)} \\
& \sum_{j=0}^{\infty} \frac{1}{j !}\left(\frac{k^{2}}{m^{2}}\right)^{j} \frac{\Gamma(a+j) \Gamma\left(a+b-\frac{d}{2}+j\right)}{\Gamma\left(\frac{d}{2}+j\right)} .
\end{aligned}
$$

What is left to do now is the sum over Gamma functions. Fortunately, there are methods developed [1112 for expanding in $\epsilon$ such hypergeometric sums in terms of objects called nested sums. Note that in the specific example above, for $d=4-2 \epsilon$ one would have integer valued coefficients in $\Gamma$-functions, while for $d=3-2 \epsilon$ one is left with half-integer valued coefficients.

\section{Nested Sums}

S-Sums [1011] are defined recursively as: $S(n)=1$ for $n>0, S(n)=0$ for $n \leq 0$ and

$$
S\left(n ; m_{1}, \ldots, m_{k} ; x_{1}, \ldots, x_{k}\right)=\sum_{i=1}^{n} \frac{x_{1}^{i}}{i^{m_{i}}} S\left(i ; m_{2}, \ldots, m_{k} ; x_{2}, \ldots, x_{k}\right) .
$$

Gamma functions can be naturally expanded in terms of S-sums, according to:

$$
\frac{\Gamma(j+1+\epsilon)}{\Gamma(1+\epsilon)}=\Gamma(j+1) \exp \left(-\sum_{k=1}^{\infty} \epsilon^{k} \frac{(-1)^{k}}{k} S(j ; k ; 1)\right) .
$$

So if we can represent Feynman integrals in terms of one infinite sum over Gamma functions, we can expand the Gamma functions in terms of S-Sums, use their algebra 10[11 to reduce products of S-sums to a linear combination of S-sums and finally do the last infinite sum over S-sums. This last sum gives S-sums with argument infinity, 
which are, per definition, multiple polylogarithms (MPL) [13. In eq.(6,7) MPL simplify further to multiple $\zeta$-values [14. There are packages for the expansion of hypergeometric functions with integer valued coefficients [15]16]17] and of some classes of functions with half-integer coefficients [18. However, for some of our Masters, in particular the one of eq.(6), these are not sufficient, what prompted us to implement the relevant algorithms of $[12$.

\section{Applications}

Here we apply the methods discussed in previous sections to two four-loop single mass scale Feynman integrals from EQCD. We first apply the Mellin-Barnes method and get hypergeometric representations. These consists in the case of eq.(7) of one hypergeometric function, which in $d=3-2 \epsilon$ is given by ${ }_{4} F_{3}\left(1,1-\epsilon, 2 \epsilon, \frac{1}{2}+\epsilon ; 2-2 \epsilon, 1+\epsilon, \frac{3}{2}-\epsilon ; 1\right)$ and in case of eq.(6) of nine different hypergeometric functions, of which the highest one is ${ }_{5} F_{4}\left(1,1-\epsilon, \frac{3}{2}+\epsilon, \frac{3}{2}+\epsilon, 2+2 \epsilon ; 2-2 \epsilon, \frac{3}{2}-\epsilon, \frac{5}{2}+\epsilon, 2+\epsilon ; 1\right)$. As mentioned above, we implemented the algorithms for expansion in $\epsilon$ of half-integer valued balanced hypergeometric functions in FORM [19]. Hypergeometric representations in $d$ dimensions and expansions in $d=4-2 \epsilon$ will be published elsewhere [20]. Note that both integrals are normalized by the appropriate power of the massive one-loop tadpole integral.

$$
\begin{aligned}
\frac{(12)^{4}=}{4} & \frac{1}{4} \zeta_{2}-\frac{1}{2} \ln ^{2} 2+\epsilon\left(-4 \zeta_{3}-\frac{5}{2} \zeta_{2}+\frac{9}{2} \ln 2 \zeta_{2}+5 \ln ^{2} 2+\ln ^{3} 2\right) \\
& +\epsilon^{2}\left(30 a_{4}+40 \zeta_{3}+13 \zeta_{2}-\frac{1}{4} \zeta_{2}^{2}-\frac{21}{4} \ln 2 \zeta_{3}-45 \ln 2 \zeta_{2}-26 \ln ^{2} 2\right. \\
& \left.-\frac{23}{2} \ln ^{2} 2 \zeta_{2}-10 \ln ^{3} 2+\frac{1}{12} \ln ^{4} 2\right)+\epsilon^{3}\left(-28 a_{5}-\frac{2103}{16} \zeta_{5}-300 a_{4}\right. \\
& -208 \zeta_{3}-54 \zeta_{2}-13 \zeta_{2} \zeta_{3}+\frac{5}{2} \zeta_{2}^{2}+28 \ln 2 a_{4}+\frac{105}{2} \ln 2 \zeta_{3}+234 \ln 2 \zeta_{2} \\
& +\frac{361}{5} \ln 2 \zeta_{2}^{2}+108 \ln ^{2} 2+\frac{213}{4} \ln ^{2} 2 \zeta_{3}+115 \ln ^{2} 2 \zeta_{2}+52 \ln ^{3} 2 \\
& \left.-\frac{14}{3} \ln ^{3} 2 \zeta_{2}-\frac{5}{6} \ln ^{4} 2+\frac{12}{5} \ln ^{5} 2\right)+\mathcal{O}\left(\epsilon^{4}\right) \\
& =\frac{7}{4 \epsilon}+7-8 \ln 2+\epsilon\left(49+16 \zeta_{2}-32 \ln 2+16 \ln ^{2} 2\right) \\
+ & \epsilon^{2}\left(308-108 \zeta_{3}+64 \zeta_{2}-224 \ln 2-64 \zeta_{2} \ln _{2} 2+64 \ln ^{2} 2-\frac{64}{3} \ln ^{3} 2\right) \\
+ & \epsilon^{3}\left(1904+128 a_{4}-432 \zeta_{3}+448 \zeta_{2}+\frac{412}{5} \zeta_{2}^{2}-1408 \ln ^{2}+544 \zeta_{3} \ln 2\right. \\
& \left.-256 \zeta_{2} \ln 2+448 \ln ^{2} 2+96 \zeta_{2} \ln 22-\frac{256}{3} \ln ^{3} 2+\frac{80}{3} \ln ^{4} 2+426 \zeta_{4}\right) \\
+ & \epsilon^{4}\left(11648+512 a_{5}-3212 \zeta_{5}+512 a_{4}-3024 \zeta_{3}+2816 \zeta_{2}-1088 \zeta_{2} \zeta_{3}\right.
\end{aligned}
$$




$$
\begin{aligned}
& +\frac{1648}{5} \zeta_{2}^{2}-8704 \ln 2+2176 \ln 2 \zeta_{3}-1792 \ln 2 \zeta_{2}-\frac{1648}{5} \ln 2 \zeta_{2}^{2} \\
& +2816 \ln ^{2} 2-1088 \ln ^{2} 2 \zeta_{3}+384 \ln ^{2} 2 \zeta_{2}-1792 / 3 \ln ^{3} 2-128 \ln ^{3} 2 \zeta_{2} \\
& \left.+\frac{320}{3} \ln ^{4} 2-64 / 3 \ln ^{5} 2+1704 \zeta_{4}-1704 \zeta_{4} \ln 2\right) \\
& +\epsilon^{5}\left(70784-256 s_{6}+2048 a_{6}+2048 a_{5}-12848 \zeta_{5}+3584 a_{4}\right. \\
& -19008 \zeta_{3}+3768 \zeta_{3}^{2}+17408 \zeta_{2}-4352 \zeta_{2} \zeta_{3}+\frac{11536}{5} \zeta_{2}^{2}+\frac{7968}{35} \zeta_{2}^{3} \\
& -53248 \ln 2+13344 \ln 2 \zeta_{5}+15232 \ln 2 \zeta_{3}-11264 \ln 2 \zeta_{2} \\
& +4352 \ln 2 \zeta_{2} \zeta_{3}-\frac{6592}{5} \ln 2 \zeta_{2}^{2}+17408 \ln ^{2} 2-4352 \ln ^{2} 2 \zeta_{3} \\
& +2688 \ln ^{2} 2 \zeta_{2}+\frac{3296}{5} \ln 22 \zeta_{2}^{2}-\frac{11264}{3} \ln ^{3} 2+\frac{4352}{3} \ln ^{3} 2 \zeta_{3}-512 \ln ^{3} 2 \zeta_{2} \\
& +\frac{2240}{3} \ln ^{4} 2+128 \ln ^{4} 2 \zeta_{2}-\frac{256}{3} \ln ^{5} 2+\frac{128}{9} \ln ^{6} 2+11928 \zeta_{4} \\
& \left.+5592 \zeta_{4} \zeta_{2}-6816 \zeta_{4} \ln 2+3408 \zeta_{4} \ln ^{2} 2+11146 \zeta_{6}\right)+\mathcal{O}\left(\epsilon^{6}\right),
\end{aligned}
$$

where $s_{6}=\sum_{i_{1}=1}^{\infty} \frac{(-1)^{i_{1}}}{i_{1}^{5}} \sum_{i_{2}=1}^{i_{1}} \frac{(-1)^{i_{2}}}{i_{2}}=0.987441 \ldots$ and $a_{i}=\operatorname{Li}_{i}\left(\frac{1}{2}\right)$.

\section{Summary}

We implemented in FORM algorithms for expanding hypergeometric functions, including expansion around half-integer balanced values. As an application we showed expansions in $d=3-2 \epsilon$ of two four-loop bubbles needed in the context of hot QCD.

\section{References}

[1] T. Appelquist and R. D. Pisarski, Phys. Rev. D 23 (1981) 2305.

[2] P. H. Ginsparg, Nucl. Phys. B 170 (1980) 388.

[3] E. Braaten and A. Nieto, Phys. Rev. D 51 (1995) 6990

[4] Y. Schröder, Nucl. Phys. Proc. Suppl. 116 (2003) 402

[5] K. G. Chetyrkin and F. V. Tkachov, Nucl. Phys. B 192 (1981) 159.

[6] F. V. Tkachov, Phys. Lett. B 100 (1981) 65.

[7] S. Laporta, Int. J. Mod. Phys. A 15 (2000) 5087

[8] E. E. Boos, A. I. Davydychev, Theor. Math. Phys. 89 (1991) 1052 [Teor. Mat. Fiz. 89 (1991) 56].

[9] V. A. Smirnov, Springer Tracts Mod. Phys. 211 (2004) 1.

[10] J. A. M. Vermaseren, Int. J. Mod. Phys. A 14, 2037 (1999)

[11] S. Moch, P. Uwer and S. Weinzierl, J. Math. Phys. 43 (2002) 3363

[12] S. Weinzierl, J. Math. Phys. 45 (2004) 2656

[13] A. B. Goncharov, Math. Res. Lett. 5497 (1998)

[14] J. M. Borwein et al., Trans. Am. Math. Soc. 353 (2001) 907

[15] S. Moch and P. Uwer, Comput. Phys. Commun. 174 (2006) 759

[16] T. Huber and D. Maitre, Comput. Phys. Commun. 175 (2006) 122

[17] S. Weinzierl, Comput. Phys. Commun. 145 (2002) 357

[18] T. Huber and D. Maitre, Comput. Phys. Commun. 178 (2008) 755

[19] J. A. M. Vermaseren, arXiv:math-ph/0010025. 
[20] E. Bejdakic and Y. Schröder, in preparation 